OPEN

SUBJECT AREAS:

CANCER GENOMICS

BIOCHEMISTRY

BIOPHYSICS

SYSTEMS BIOLOGY

Received

16 April 2013

Accepted

30 September 2013

Published

17 October 2013

Correspondence and requests for materials should be addressed to

J.J.Z. (Jie.Zheng@ stjude.org)

\section{Genome-wide network analysis of Wnt signaling in three pediatric cancers}

\author{
Ju Bao, Ho-Jin Lee \& Jie J. Zheng
}

Department of Structural Biology, St. Jude Children's Research Hospital, Memphis, TN 38105.

Genomic structural alteration is common in pediatric cancers, and analysis of data generated by the Pediatric Cancer Genome Project reveals such tumor-related alterations in many Wnt signaling-associated genes. Most pediatric cancers are thought to arise within developing tissues that undergo substantial expansion during early organ formation, growth and maturation, and Wnt signaling plays an important role in this development. We examined three pediatric tumors-medullobastoma, early T-cell precursor acute lymphoblastic leukemia, and retinoblastoma-that show multiple genomic structural variations within Wnt signaling pathways. We mathematically modeled this pathway to investigate the effects of cancer-related structural variations on Wnt signaling. Surprisingly, we found that an outcome measure of canonical Wnt signaling was consistently similar in matched cancer cells and normal cells, even in the context of different cancers, different mutations, and different Wnt-related genes. Our results suggest that the cancer cells maintain a normal level of Wnt signaling by developing multiple mutations.

urrent international efforts to identify oncogenic somatic mutations have revealed that not only single
nucleotide variations but also genomic structural variations (SVs) play a key role in human cancers ${ }^{1,2}$. In nucleotide variations but also genomic structural variations (SVs) play a key role in human cancers ${ }^{1,2}$. In
fact, SVs are the main mutations that drive many pediatric cancers ${ }^{3-6}$. Given the immense complexity of some structural alterations, the full set of SVs implicated in oncogenesis would best be identified from wholegenome sequencing data ${ }^{7,8}$. By mapping partial reads to a reference genome, the newly developed CREST (Clipping Reveals Structure) algorithms were able to identify reads spanning the junctions of SVs with a validation rate greater than $80 \%$. Using data from the current St. Jude Children's Research Hospital-Washington University Pediatric Cancer Genome Project (PCGP) ${ }^{3}$, such approach has identified many SVs in medulloblastoma $(\mathrm{MB})^{6}$, early T-cell precursor acute lymphoblastic leukemia (T-ALL) ${ }^{4}$, and retinoblastoma (RB) ${ }^{9}$. Examining the SVs in the three cancers, we observed a very high frequency of somatic SVs in canonical Wnt signaling-associated genes: $41.3 \%$ in T-ALL (38/92 patients), 50\% in RB (2/4 patients), and 61.8\% in MB (47/76 patients). However, within even a single type of cancer, SVs spanned almost the entire canonical Wnt signaling pathway. Further, most cases had multiple Wnt gene structural mutations (Fig. 1a). Therefore, a systems biology analysis equipped by a mathematical model is needed to investigate the consequences of these mutations and their effect on the Wnt signaling pathway in individual patients ${ }^{10}$.

\section{Results}

Because dysregulation of the canonical Wnt signaling pathway can cause aberrant cell proliferation and chromosome instability, the canonical pathway has been extensively studied ${ }^{11,12}$. In the absence of Wnt signaling, axin, APC, and GSK-3 $\beta$ form a complex that phosphorylates $\beta$-catenin, targeting it for proteasomal degradation. Canonical Wnt signaling is activated by the binding of Wnt protein to its co-receptors Frizzled (FzD) and LRP5/6. The dimerization of these two receptors transduces Wnt signal into the cell, destroying axin/APC/GSK-3 $\beta$ and stabilizing cytoplasmic $\beta$-catenin. As a consequence, $\beta$-catenin translocates to the nucleus where, in association with TCF, it activates Wnt target genes ${ }^{13}$.

Lee et $\mathrm{al}^{14}$ first used a mathematical model to describe the canonical Wnt signaling pathway. The model has since been extended to include additional protein-protein interactions ${ }^{15-18}$. Moreover, the model recently presented by Kogan et $\mathrm{al}^{19}$ explicitly describes extracellular signal initiation and regulatory interactions of two major Wnt signaling antagonists, Dickkopf $(\mathrm{DKK})$ and soluble frizzled-related protein (sFRP) ${ }^{20,21}$. To incorporate as many elements of the Wnt pathway as possible, we integrated several existing models to form a single, comprehensive mathematical model of the canonical Wnt signaling pathway containing 14 ordinary differential equations (ODEs) and 24 variables (14 independent and 10 dependent) that explicitly represent nearly all major interactions and components of the Wnt pathway. Our model, which has been validated (Supplementary Fig. S2) 


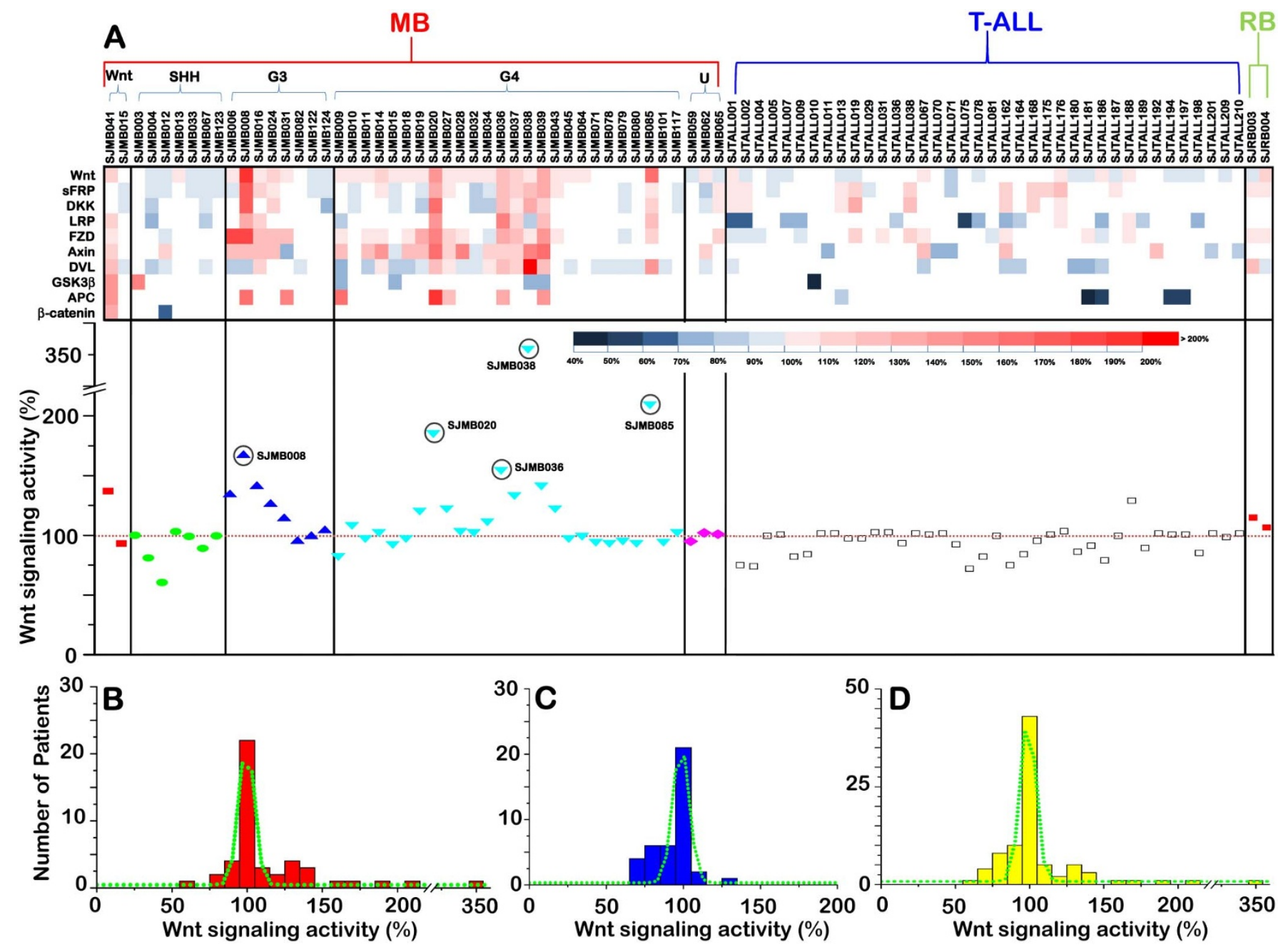

Figure 1 Comparison of Wnt signaling output in matched cancer cells and normal cells. (A) $\beta$-catenin levels in cancer cells vs. normal cells according to cancer type and genomic SV. SVs (equivalent to copy number variations) in Wnt signaling-associated genes in MB, ALL, and RB patients studied in the PCGP are listed in the upper panel. There are five subgroups (Wnt, SHH, group 3, group 4, and unclassified) within MB. In the lower panel, the calculated $\beta$-catenin level is shown as a percentage of the $\beta$-catenin level in matched normal cells. (B) Distribution of $\beta$-catenin levels in MB cells with SVs in Wnt signaling genes. (C) Distribution of $\beta$-catenin levels in T-ALL cells with SVs in Wnt signaling genes. (D) Distribution of $\beta$-catenin levels in the malignant cells of all studied patients (the MB, TALL, and RB tumor groups). Values in panels b, $c$, and $\mathrm{d}$ are the number of cases within a range of 10 percentage points. The green lines are the fitted Gaussian distributions.

by comparison with others ${ }^{14,19}$, uses the intracellular $\beta$-catenin level as a measure of Wnt signaling outcome (also termed Wnt signaling output). Parameter sensitivity was analyzed as described by Goentoro et $\mathrm{al}^{22}$. We found that the parameters most sensitive to perturbation were those associated with Dishevelled (Dvl); a 5-fold increase in those parameters resulted in a slightly greater than 3-fold increase in Wnt signaling output. Nevertheless, the change in Wnt signaling outcome was, overall, relatively insensitive to the perturbation of individual Wnt signaling-associated genes (Supplementary Figure S3). This result is consistent with analysis using the model described by Lee at $\mathrm{al}^{14}$.

Using our mathematical model, based on a notion that globally there is indeed an appreciable correlation between mRNA levels and gene copy number ${ }^{23}$, we examined how SVs in cancer cells affect the outcome of Wnt signaling by comparing $\beta$-catenin levels (i.e., Wnt signaling outcomes) in cancer cells carrying SVs to those in normal cells from the same PCGP patient. An increasing concentration of Wnt ligands induces a progressively increasing cellular $\beta$-catenin level until $\beta$-catenin reaches a plateau ${ }^{14,22}$, and both sFRP and DKK inhibit this progressive accumulation of $\beta$-catenin. To maximize the sensitivity of the comparisons, we elected to compare $\beta$-catenin levels in the presence of $0.25 \mathrm{nM} \mathrm{Wnt,} 1 \mathrm{nM}$ sFRP, and $0.5 \mathrm{nM} \mathrm{DKK}$, as these concentrations are consistent with the midpoint of $\beta$-catenin accumulation (i.e., half of the saturated $\beta$-catenin level) and are most sensitive to variations in other Wnt signaling parameters. $\beta$-catenin levels in malignant cells were converted to Wnt signaling output as a percentage of $\beta$-catenin levels in matched normal cells (assigned a value of $100 \%$ ) (Fig. 1A, Supplementary Table S4).

Surprisingly, $\beta$-catenin levels in most patients' cancer cells were within approximately $10 \%$ of levels in germline cells; in all three cancer types, $\beta$-catenin levels in cancer cells showed a Gaussian distribution, peaking near the peak levels in normal cells. In patients with $\mathrm{MB}$, the mean peak $\beta$-catenin level in malignant cells was 99.59\% of that in normal cells, and the Gaussian distribution width of the cancer-cell $\beta$-catenin level was $10.09 \%$ that of the germline-cell $\beta$-catenin level (Fig. 1B). $\beta$-catenin levels were similarly distributed in T-ALL patients' cancer cells; the mean peak $\beta$-catenin level in cancer cells was $98.42 \%$ of that in normal cells and the Gaussian distribution width was $10.38 \%$ of that in germline cells (Fig. 1C). When the three types of cancers were combined, the overall mean 
peak $\beta$-catenin level was $98.98 \%$ of that in normal cells and the Gaussian distribution width of $\beta$-catenin levels in cancer cells was $12.28 \%$ of that in germline cells (Fig. 1D).

\section{Discussion}

The results of our computational studies suggest that canonical Wnt signaling activity is normally distributed in cancer cells and has a mean value approximating that in normal cells. The very few outlier values we observed may reflect the limitations of our mathematical model, which is the most comprehensive model of canonical Wnt signaling to date but is unlikely to account for all components of the pathway. For example, Dvl expression was substantially upregulated in four of the five cases of MB (SJMB020, SJMB036,SJMB038, and SJMB085) that had unusually high $\beta$-catenin values (Fig. 1a). Dvl is well documented to have many binding partners ${ }^{24-26}$, the identities of which are only partially known ${ }^{27}$. Therefore, high Wnt signaling output in these four cases may reflect the absence of Dvl regulators from our model. In addition, our model lacks integrated feedback loops ${ }^{28}$. Such an omission may also have introduced calculation errors that account for outlier values.

Canonical Wnt signaling is a key mechanism in tissue development. Because dysregulation of tissue development is thought to be linked to oncogenesis in children, it was not surprising that almost half the tumors we examined showed a large number of mutations. What was surprising, however, were the similar levels of Wnt signaling (i.e., levels of $\beta$-catenin) in cancer cells and in the corresponding germline cells in most of the studied tumors, despite multiple mutations in the Wnt pathway. Our data suggest that during development, tumor cells generate multiple mutations to maintain normal Wnt signaling output under environmental pressure. Indeed, like other signaling pathways ${ }^{28}$, canonical Wnt signaling pathway has feedback loops. For example, in the pathway, Axin functions as an inhibition factor; and it was reported that Axin2 is a direct target of the pathway $^{29}$. In the three pediatric cancers we examined, we found that 20 patients had upregulation of Axin2 (Supplementary Table S5). Interestingly, 12 out of the 20 had very high Wnt signaling outcome. The 12 cases actually represented almost all the patients who had high Wnt signaling outcome in our study (Figure 1A, Supplementary Tables S4 and S5). Moreover, within the 12, except one outliner, there is a clear linear correlation between Axin2 SVs and Wnt signaling outcomes (Supplementary Figure S4) suggesting a likely feedback mechanism. Therefore, these mutations in the Wnt signaling pathway are unlikely to act as drivers of the primary cancer in these patients. This conclusion is consistent with a previous report that $\beta$-catenin staining of ALL samples suggested no upregulation of canonical Wnt signaling ${ }^{30}$. In addition, abnormal $\beta$-catenin immunoreactivity was absent from MB samples except in the Wnt subgroup $^{31}$. Moreover, in our study only 2 of 12 patients in this Wnt subgroup had SVs in canonical Wnt signaling-associated genes, while tumor cells in 8 of the 12 patients expressed $\beta$-catenin-stabilizing mutants ${ }^{6}$. Interestingly, many of these cases also revealed missense mutations in the DEAD-box RNA helicase DDX3X at chromosome Xp11.3. Because DDX3 is reported to promote Wnt signaling $^{32}$, it is likely that these missense mutations balance the effects of the $\beta$-catenin-stabilizing mutants in the tumor cells.

Even when normal Wnt signaling output is maintained in tumor cells via abnormal Wnt signaling pathways, these Wnt pathways are clearly much less stable than those in normal cells. For example, when we artificially activated Wnt signaling in our model, some cancer cells showed much higher Wnt activity than did normal cells (Supplementary Figure S5A). Therefore, when metastatic tumor cells reach a new environment, their Wnt signaling output may not be maintained. In such a case, it is very possible that abnormal Wnt signaling becomes a cancer driver in the new site. On the other hand, our finding of similar Wnt signaling outcome in malignant and germline tissue despite multiple Wnt pathway mutations provides a novel opportunity for targeted therapy for the three pediatric cancers examined. Due to the mutations, the Wnt signaling pathways in tumor and germline tissue were not identical, despite their similar $\beta$-catenin output. Therefore, an inhibitor that targets a specific protein-protein interaction within the pathway may disrupt abnormal Wnt signaling in tumor cells while sparing Wnt signaling in normal cells, thereby disrupting tumor growth while maintaining normal tissue development. Many small-molecule Wnt signaling regulators, inhibitors, and activators that target different proteinprotein interactions have been developed and characterized ${ }^{33,34}$; and our calculations indicate that they have different effects on Wnt signaling in cancer cells in different patients (Supplementary Figure S5B). Therefore, a systems biology analysis using a mathematical model like the one described here should be able to use the mutations revealed by whole-genome sequencing to identify a small-molecule regulator or combination of regulators that most effectively disrupts cancer cell growth while maximally sparing normal cells $s^{10,35}$. Making use of such therapeutic opportunities may represent the future of genome-based personalized medicine.

\section{Methods}

Mathematical model. Our model explicitly represents each known major component of the canonical Wnt signaling pathway. As shown in Supplementary Figure S1, this model focuses on the following events: a) Extracellular activation of Wnt signaling by binding of Wnt protein to FzD and LRP, and antagonism of Wnt signaling by competitive binding of FRP and DKK to FzD and LRP, respectively; b) Activation of Dvl by the Wnt-LRP-FzD ternary complex; c) Deactivation by Dvl of the APC-axinGSK destruction complex, which binds to $\beta$-catenin and results in $\beta$-catenin phosphorylation and degradation, thereby stabilizing $\beta$-catenin levels.

Using the assumption of well-mixing and the law of mass action for all protein interactions in our model, we rewrote the key extracellular and membrane interactions into 7 ODEs and combined them with the original 7 ODEs of Lee et $\mathrm{al}^{14}$ by replacing the Wnt concentration with the Wnt-LRP-Fzd complex concentration (Supplementary Table S1). This model contains 24 variables (14 independent and 10 dependent variables) (Supplementary Table S2). The partition coefficient Ksu-ex introduced by Kogan et $\mathrm{al}^{19}$, which transforms the values of the signals in the cell membrane to extracellular concentrations, was retained to compute reactions between components measured by different units in extracellular solution and membrane. The rate of each reaction and the original concentration of each protein were obtained directly from the parameters of Kogan et $\mathrm{al}^{19}$ for modeling of extracellular and membrane reactions and the parameters of Lee et $\mathrm{al}^{14}$ for intracellular reactions (Supplementary Table S3). The model was solved numerically by the ODE solver ODE15s in the MATLAB software package. The Wnt signaling pathway contains 16 Wnt subtypes, 10 Frizzled subtypes, 2 LRP subtypes, 5 sFRP subtypes, 3 DKK subtypes (DKK3 is not involved in Wnt signaling), 3 Dvl subtypes, and two axin subtypes. Each protein subtype was assumed to have the same kinetic parameters and the same physiological concentration.

Model validation. Our model was compared with the models reported by Lee et $\mathrm{al}^{14}$ and Kogan et $\mathrm{al}^{19}$. The $\beta$-catenin levels were calculated as the functions of the Wnt concentration in the three models, respectively. In general, $\beta$-catenin accumulation profiles generated by the three models were consistent. Because the valid Wnt concentration range for modeling studies was suggested to be $0 \mathrm{nM}$ to $1.4 \mathrm{nM}^{14}$, all of the simulations were performed within this range. The calculated Wnt dosedependent activation results showed that the $\beta$-catenin level increases rapidly as Wnt concentration rises from $0 \mathrm{nM}$ to $0.5 \mathrm{nM}$, then reaches its saturation point

(Supplementary Fig. S2A), consistent with the profile generated by the model of Lee et $\mathrm{al}^{14}$. The profile generated by the model of Kogan et $\mathrm{al}^{19}$ reaches saturation at a slightly lower Wnt concentration.

Using our model, we studied the inhibitory effects of sFRP and DKK at a fixed concentration $(0.5 \mathrm{nM})$ of Wnt (Supplementary Figure S2B, C). The calculated $\mathrm{IC}_{50}$ values were $11 \mathrm{nM}$ for $\mathrm{sFRP}$ and $1.8 \mathrm{nM}$ for DKK1, closely approximating the results obtained by using the model of Kogan et $\mathrm{al}^{19}$ and within the ranges of the experimentally determined $\mathrm{IC}_{50} \mathrm{~s}$ of the two inhibitors ${ }^{20,21}$. We also examined the combinatorial inhibitory effects of adding both sFRP and DKK, and the resulting $\beta$-catenin concentrations in cells agreed well with that calculated by using the model of Kogan et $\mathrm{al}^{19}$ (Supplementary Figure S1D). These results confirmed that our model is fully compatible with the two existing models within a Wnt concentration range of 0 to $1.4 \mathrm{nM}$.

Wnt signaling output in cancer cells. Our mathematical model was used to examine the effect of SVs of Wnt signaling pathway elements on Wnt signaling outcomes in the cancer cells of PCGP patients. We used cellular $\beta$-catenin concentration as the measure of Wnt signaling outcome/output and calculated $\beta$-catenin levels in cancer cells as a percentage of levels in matched germline cells. As the concentration of Wnt ligands increases, the concentration of cellular $\beta$-catenin also increases until it reaches a plateau ${ }^{14,22}$, and both sFRP and DKK inhibit the accumulation of $\beta$-catenin induced 
by Wnt signaling. To maximize the sensitivity of comparisons, we compared $\beta$ catenin levels in the presence of $0.25 \mathrm{nM}$ Wnt, consistent with the midpoint of $\beta$ catenin accumulation (i.e., in the presence of $0.25 \mathrm{nM} \mathrm{Wnt}$, the level of $\beta$-catenin is at half the saturated level); this concentration is most sensitive to variations in other parameters of the Wnt signaling pathway (Supplementary Figure S2A). Under these conditions, the $\beta$-catenin levels in cancer cells with Wnt signaling gene variations were calculated and expressed as a percentage of the $\beta$-catenin levels in normal cells (assigned a value of $100 \%$ ). Various physiological concentrations of sFRP and DKK have been reported ${ }^{20,21}$; on the basis of our computational studies of their inhibitory effects, we chose reference concentrations of $1 \mathrm{nM}$ sFRP and $0.5 \mathrm{nM}$ DKK, the concentrations at which the inhibitory effects of the two components are most sensitive to changes in their concentration (Supplementary Figure S2B-D). Other parameters were obtained from published reports. Supplementary Table S4 details the variations in expression of genes in the Wnt signaling pathway in the studied patients.

Using our model, we computed the Wnt signaling output of the cancer cells and normal cells of individual PCGP patients. Wnt output in cancer cells was expressed as a percentage of output in the respective matched normal cells; the data are shown in Figure 1 and are listed in Supplementary Table S4.

1. Beroukhim, R. et al. The landscape of somatic copy-number alteration across human cancers. Nature 463, 899-905 (2010).

2. Inaki, K. \& Liu, E. T. Structural mutations in cancer: mechanistic and functional insights. Trends Genet 28, 550-9 (2012).

3. Downing, J. R. et al. The Pediatric Cancer Genome Project. Nat Genet 44, 619-22 (2012)

4. Zhang, J. et al. The genetic basis of early T-cell precursor acute lymphoblastic leukaemia. Nature 481, 157-63 (2012).

5. Northcott, P. A. et al. Subgroup-specific structural variation across 1,000 medulloblastoma genomes. Nature 488, 49-56 (2012).

6. Robinson, G. et al. Novel mutations target distinct subgroups of medulloblastoma. Nature 488, 43-8 (2012).

7. Medvedev, P., Stanciu, M. \& Brudno, M. Computational methods for discovering structural variation with next-generation sequencing. Nat Methods 6, S13-20 (2009)

8. Wang, J. et al. CREST maps somatic structural variation in cancer genomes with base-pair resolution. Nat Methods 8, 652-4 (2011).

9. Zhang, J. et al. A novel retinoblastoma therapy from genomic and epigenetic analyses. Nature 481, 329-34 (2012).

10. Aldridge, B. B., Burke, J. M., Lauffenburger, D. A. \& Sorger, P. K. Physicochemical modelling of cell signalling pathways. Nat Cell Biol 8, 1195-203 (2006).

11. Polakis, P. Wnt signaling in cancer. Cold Spring Harb Perspect Biol 4 (2012).

12. van Amerongen, R. \& Nusse, R. Towards an integrated view of Wnt signaling in development. Development 136, 3205-14 (2009).

13. Cadigan, K. M. Wnt/beta-catenin signaling: turning the switch. Dev Cell 14, 322-3 (2008)

14. Lee, E., Salic, A., Kruger, R., Heinrich, R. \& Kirschner, M. W. The roles of APC and Axin derived from experimental and theoretical analysis of the Wnt pathway. PLoS Biol 1, E10 (2003).

15. Kim, D., Rath, O., Kolch, W. \& Cho, K. H. A hidden oncogenic positive feedback loop caused by crosstalk between Wnt and ERK pathways. Oncogene 26, 4571-9 (2007).

16. Mirams, G. R., Byrne, H. M. \& King, J. R. A multiple timescale analysis of a mathematical model of the Wnt/beta-catenin signalling pathway. J Math Biol 60, 131-60 (2010)

17. Shin, S. Y. et al. Functional roles of multiple feedback loops in extracellular signalregulated kinase and Wnt signaling pathways that regulate epithelialmesenchymal transition. Cancer Res 70, 6715-24 (2010)

18. Wawra, C., Kuhl, M. \& Kestler, H. A. Extended analyses of the Wnt/beta-catenin pathway: robustness and oscillatory behaviour. FEBS Lett 581, 4043-8 (2007).

19. Kogan, Y. et al. A new validated mathematical model of the Wnt signalling pathway predicts effective combinational therapy by sFRP and Dkk. Biochem 444, 115-25 (2012).
20. Kawano, Y. \& Kypta, R. Secreted antagonists of the Wnt signalling pathway. J Cell Sci 116, 2627-34 (2003)

21. Bao, J., Zheng, J. J. \& Wu, D. The Structural Basis of DKK-Mediated Inhibition of Wnt/LRP Signaling. Sci Signal 5, pe22 (2012).

22. Goentoro, L. \& Kirschner, M. W. Evidence that fold-change, and not absolute level, of beta-catenin dictates Wnt signaling. Mol Cell 36, 872-84 (2009).

23. Weischenfeldt, J., Symmons, O., Spitz, F. \& Korbel, J. O. Phenotypic impact of genomic structural variation: insights from and for human disease. Nat Rev Genet 14, 125-138 (2013)

24. Wharton, K. A., Jr. Runnin' with the Dvl: proteins that associate with Dsh/Dvl and their significance to Wnt signal transduction. Dev.Biol. 253, 1-17 (2003).

25. Wallingford, J. B. \& Habas, R. The developmental biology of Dishevelled: an enigmatic protein governing cell fate and cell polarity. Development 132, 4421-4436 (2005).

26. Gao, C. \& Chen, Y. G. Dishevelled: The hub of Wnt signaling. Cell Signal 22, 717-27 (2009).

27. Lee, H. J. et al. Identification of transmembrane protein 88 (TMEM88) as a dishevelled-binding protein. J Biol Chem 285, 41549-56 (2010).

28. Cloutier, M. \& Wang, E. Dynamic modeling and analysis of cancer cellular network motifs. Integr Biol (Camb) 3, 724-32 (2011).

29. Jho, E. H. et al. Wnt/beta-catenin/Tcf signaling induces the transcription of Axin2, a negative regulator of the signaling pathway. Mol Cell Biol 22, 1172-83 (2002).

30. Serinsoz, E. et al. Aberrant expression of beta-catenin discriminates acute myeloid leukaemia from acute lymphoblastic leukaemia. Br J Haematol 126, 313-9 (2004).

31. Ellison, D. W. et al. Medulloblastoma: clinicopathological correlates of $\mathrm{SHH}$, WNT, and non-SHH/WNT molecular subgroups. Acta Neuropathol 121, 381-96 (2011).

32. Cruciat, C. M. et al. RNA helicase DDX3 is a regulatory subunit of casein kinase 1 in Wnt-beta-catenin signaling. Science 339, 1436-41 (2013).

33. Barker, N. \& Clevers, H. Mining the Wnt pathway for cancer therapeutics. Nat.Rev.Drug Discov. 5, 997-1014 (2006).

34. Polakis, P. Drugging Wnt signalling in cancer. EMBO J 31, 2737-46 (2012)

35. Saez-Rodriguez, J. et al. Discrete logic modelling as a means to link protein signalling networks with functional analysis of mammalian signal transduction. Mol Syst Biol 5, 331 (2009).

\section{Acknowledgements}

This work was supported by NIH grants CA21765 (Cancer Center Support Grant) and GM081492 and by the American Lebanese Syrian Associated Charities (ALSAC). We thank Drs. Tanja Gruber and Amar Gajjar for critical reading of the manuscript and Sharon Naron for editing the manuscript.

\section{Author contributions}

All authors contributed to analysis and interpretation of the data and writing of the manuscript. J.B. generated the mathematical model. H.-J.L. acquired and summarized the data. J.J.Z. conceived, designed and supervised the studies.

\section{Additional information}

Supplementary information accompanies this paper at http://www.nature.com/ scientificreports

Competing financial interests: The authors declare no competing financial interests.

How to cite this article: Bao, J., Lee, H.-J. \& Zheng, J.J. Genome-wide network analysis of Wnt signaling in three pediatric cancers. Sci. Rep. 3, 2969; DOI:10.1038/srep02969 (2013).

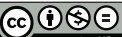

This work is licensed under a Creative Commons AttributionNonCommercial-NoDerivs 3.0 Unported license. To view a copy of this license, visit http://creativecommons.org/licenses/by-nc-nd/3.0 